\title{
Do Roux-en-Y gastric bypass patients meet the dietary guidelines?
}

Ina Gesquiere ${ }^{{ }^{*}}$, Kelly Van Meerbeeck ${ }^{2}$, Veerle Foulon ${ }^{1}$, Patrick Augustijns', Matthias Lannoo ${ }^{3}$, Ann Meulemans $^{4}$, Bart Van der Schueren ${ }^{4}$, Christophe Matthys ${ }^{4}$

From Genes and nutrition, is personalised nutrition the next realistic step?

Brussels, Belgium. 25 April 2014

\section{Background}

The prevalence of obesity has increased to epidemic proportions and, as a result, the number of bariatric surgeries has increased worldwide. To date, bariatric surgery remains the sole medical intervention that achieves considerable and sustained weight loss. As both obesity and bariatric surgery are associated with nutritional deficiencies, the aim of this study was to evaluate the dietary intake of macro- and micronutrients in patients before and after Roux-en-Y gastric bypass (RYGB).

\section{Methods}

A prospective observational study was performed at University Hospitals Leuven, Belgium. Patients com-

Table 1 Intake of macronutrients at different time-points, shown as mean \pm SD.

\begin{tabular}{lcccc}
\hline $\mathbf{n = 2 2}$ & Intake pre-RYGB & Intake $\mathbf{1}$ month post-RYGB & Intake $\mathbf{3}$ months post-RYGB & Significance \\
\hline Carbohydrates $\mathbf{( g )}$ & $245.2 \pm 72.4$ & $81.8 \pm 39.1$ & $110.9 \pm 51.42$ & 1,2 \\
\hline Proteins $\mathbf{( g )}$ & $87.3 \pm 23.8$ & $37.2 \pm 16.6$ & $48.0 \pm 14.4$ & \\
\hline Fat $\mathbf{g})$ & $92.2 \pm 40.4$ & $20.5 \pm 12.6$ & $36.3 \pm 16.2$ & $1,2,3$ \\
\hline
\end{tabular}

1 p<0.01:pre-op vs post-op 1 month; 2 p<0.01:pre-op vs post-op 3 months; 3 p<0.01:post-op 1 month vs post-op 3 months

Table 2 Intake of micronutrients at different time-points, shown as mean \pm SD.

\begin{tabular}{|c|c|c|c|c|}
\hline & $\begin{array}{c}\text { Intake pre-RYGB (32 } \\
\text { patients) }\end{array}$ & $\begin{array}{c}\text { Intake } 1 \text { month post-RYGB (28 } \\
\text { patients) }\end{array}$ & $\begin{array}{c}\text { Intake } 3 \text { months post-RYGB ( } 26 \\
\text { patients) }\end{array}$ & Significance \\
\hline $\mathrm{Ca}(\mathrm{mg})$ & $970.4 \pm 519.6$ & $638.4 \pm 287.9$ & $695.1 \pm 352.3$ & \\
\hline $\mathrm{Fe}(\mathrm{mg})$ & $12.6 \pm 3.7$ & $5 \pm 2.9$ & $6.0 \pm 1.8$ & 1,2 \\
\hline $\mathrm{Cu}(\mathrm{mg})$ & $2.1 \pm 1.5$ & $1.0 \pm 0.9$ & $4.9 \pm 18.6$ & \\
\hline $\mathrm{Zn}$ (mg) & $46.6 \pm 92.1$ & $10.2 \pm 21.1$ & $6.6 \pm 3.7$ & \\
\hline Vitamin A $(\mu \mathrm{g})$ & $962.8 \pm 405.2$ & $721.5 \pm 490.0$ & $787.5 \pm 716.6$ & \\
\hline $\begin{array}{l}\text { Vitamin B1 } \\
(\mathrm{mg})\end{array}$ & $1.7 \pm 0.7$ & $0.6 \pm 0.3$ & $0.8 \pm 0.3$ & 1,2 \\
\hline $\begin{array}{l}\text { Vitamin B12 } \\
(\mu \mathrm{g})\end{array}$ & $5.4 \pm 2.5$ & $2.3 \pm 1.5$ & $3.3 \pm 1.8$ & 1,2 \\
\hline Vitamin C (mg) & $138.9 \pm 83.8$ & $70.3 \pm 56.7$ & $85.1 \pm 52.2$ & 1,2 \\
\hline Vitamin $D(\mu \mathrm{g})$ & $8.4 \pm 5.1$ & $5.2 \pm 3.3$ & $4.2 \pm 3.2$ & \\
\hline
\end{tabular}

1 p<0.01:pre-op vs post-op 1 month; 2 p<0.01:pre-op vs post-op 3 months; 3 p<0.01:post-op 1 month vs post-op 3 months

\footnotetext{
* Correspondence: ina.gesquiere@pharm.kuleuven.be

'Department of Pharmaceutical and Pharmacological Sciences, KU Leuven,

Leuven, Belgium

Full list of author information is available at the end of the article
} 
pleted a dietary record of two non-consecutive days before RYGB and 1 and 3 months after RYGB. Intake of macronutrients and micronutrients was calculated for the different time-points. Paired sample t-tests were performed to analyse differences between time-points.

\section{Results}

\section{Conclusions}

The intake of macro- and micronutrients is markedly decreased one month after RYGB. At three months post-surgery, the intake of macronutrient increases, but the micronutrient intake remains identical at a worryingly low level. Our data clearly suggest that nutritional guidance is essential following bariatric surgery.

\section{Trial registration}

Clinicaltrials.gov \#NCT01571180.

\section{Acknowledgements}

I.G. receives a PhD scholarship from the Agency for Innovation by Science and Technology, Flanders.

\section{Authors' details}

'Department of Pharmaceutical and Pharmacological Sciences, KU Leuven, Leuven, Belgium. ${ }^{2}$ Department of Health and Technology, Leuven University College, Leuven, Belgium. ${ }^{3}$ Department of Pathology Abdominal Surgery, University Hospitals Leuven, Leuven, Belgium. ${ }^{4} \mathrm{Clinical}$ and Experimental Endocrinology, KU Leuven/University Hospitals Leuven, Leuven, Belgium.

Published: 6 June 2014

doi:10.1186/2049-3258-72-S1-P4

Cite this article as: Gesquiere et al:: Do Roux-en-Y gastric bypass patients meet the dietary guidelines? Archives of Public Health 2014 72(Suppl 1):P4.
Submit your next manuscript to BioMed Central and take full advantage of:

- Convenient online submission

- Thorough peer review

- No space constraints or color figure charges

- Immediate publication on acceptance

- Inclusion in PubMed, CAS, Scopus and Google Scholar

- Research which is freely available for redistribution

Submit your manuscript at www biomedcentral.com/submit 